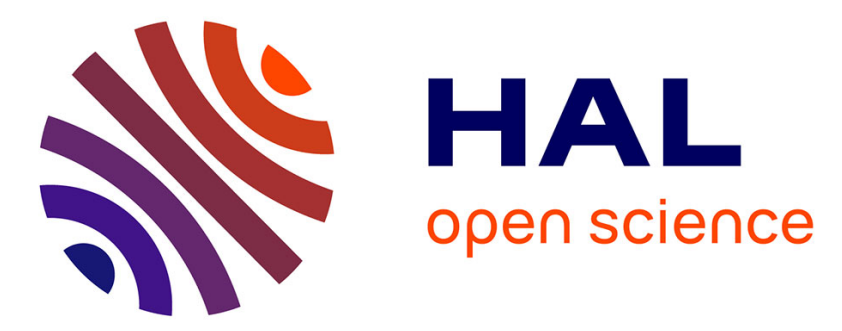

\title{
Comparison of Spectra in Unsequenced Species
}

Freddy Cliquet, Guillaume Fertin, Irena Rusu, Dominique Tessier

\section{To cite this version:}

Freddy Cliquet, Guillaume Fertin, Irena Rusu, Dominique Tessier. Comparison of Spectra in Unsequenced Species. 4th Brazilian Symposium on Bioinformatics (BSB 2009), 2009, Porto Alegre, Brazil. pp.24-35, 10.1007/978-3-642-03223-3_3 . hal-00416462

\section{HAL Id: hal-00416462 \\ https://hal.science/hal-00416462}

Submitted on 14 Sep 2009

HAL is a multi-disciplinary open access archive for the deposit and dissemination of scientific research documents, whether they are published or not. The documents may come from teaching and research institutions in France or abroad, or from public or private research centers.
L'archive ouverte pluridisciplinaire HAL, est destinée au dépôt et à la diffusion de documents scientifiques de niveau recherche, publiés ou non, émanant des établissements d'enseignement et de recherche français ou étrangers, des laboratoires publics ou privés. 


\title{
Comparison of Spectra in Unsequenced Species
}

\author{
Freddy Cliquet ${ }^{1,2}$, Guillaume Fertin ${ }^{1}$, Irena Rusu ${ }^{1}$,Dominique Tessier ${ }^{2}$ \\ 1 LINA, UMR CNRS 6241 Université de Nantes, \\ 2 rue de la Houssinière, 44322, Nantes, Cedex 03, France \\ \{freddy.cliquet, guillaume.fertin, irena.rusu\}@univ-nantes.fr \\ 2 UR1268 BIA, INRA, Rue de la Géraudière, BP 71627, 44316 Nantes, France \\ dominique.tessier@nantes.inra.fr
}

\begin{abstract}
We introduce a new algorithm for the mass spectrometric identification of proteins. Experimental spectra obtained by tandem MS/MS are directly compared to theoretical spectra generated from proteins of evolutionarily closely related organisms. This work is motivated by the need of a method that allows the identification of proteins of unsequenced species against a database containing proteins of related organisms. The idea is that matching spectra of unknown peptides to very similar MS/MS spectra generated from this database of annotated proteins can lead to annotate unknown proteins. This process is similar to ortholog annotation in protein sequence databases. The difficulty with such an approach is that two similar peptides, even with just one modification (i.e. insertion, deletion or substitution of one or several amino $\operatorname{acid}(\mathrm{s}))$ between them, usually generate very dissimilar spectra. In this paper, we present a new dynamic programming based algorithm: PacketSpectralAlignment. Our algorithm is tolerant to modifications and fully exploits two important properties that are usually not considered: the notion of inner symmetry, a relation linking pairs of spectrum peaks, and the notion of packet inside each spectrum to keep related peaks together. Our algorithm, PacketSpectralAlignment is then compared to SpectralAlignment [1] on a dataset of simulated spectra. Our tests show that PacketSpectralAlignment behaves better, in terms of results and execution time.
\end{abstract}

\section{Introduction}

In proteomics, tandem mass spectrometry (MS/MS) is a general method used to identify proteins. At first, during the MS/MS process, the peptides issued from the digestion of an unknown protein by an enzyme are ionized so that their mass may be measured. Then, the mass spectrometer isolates each peptide and fragments it into smaller ions, before measuring the corresponding masses. This process provides for each peptide an experimental spectrum in the form of a series of peaks, each peak corresponding to a mass that has been measured. From these experimental spectra, we aim at retrieving the corresponding peptide sequences. Then, by combining the peptide sequences from different spectra, our goal is to relate the unknown protein to one of the proteins stored in a protein 
database such as SwissProt. Given an experimental spectrum, there are two main possibilities to obtain the corresponding peptide sequence: de novo sequencing or spectra comparison.

De novo: In de novo sequencing, the spectrum is directly interpreted and a corresponding amino acid sequence of the peptide is inferred without using any other data than the spectrum itself. Currently, the analysis of unsequenced species is mainly done by de novo interpretation $[2,3]$. The main reason is the speed of each interpretation and the fact that the peptide sequence does not need to be part of a protein database. An important drawback is that this method requires high quality spectra and still leads to a lot of interpretation errors $[4,5]$.

Spectra Comparison: In spectra comparison, an experimental spectrum is compared with theoretically predicted spectra. The theoretical spectra are inferred from the different peptides generated by an in-silico digestion of all the proteins contained in a database. A score function is used to evaluate each possible comparison, and the results are ordered according to this score. The theoretical spectrum with the best score is associated to the corresponding experimental spectrum. There are a number of existing softwares that match uninterpreted MS/MS experimental spectra to theoretical spectra, such as SEQUEST [6] or MASCOT [7]. They are based on the Shared Peaks Count (SPC) algorithm, an algorithm that simply counts the number of peaks in common between the two spectra, but presents zero tolerance to modifications (i.e. insertion, deletion or substitution of one or several amino acid(s)). This is due to the fact that the slightest modification in a peptide sequence highly changes the contents of the corresponding spectrum, and thus can lead to false identifications. Two approaches have already been explored to take modified peptides into account. The first one consists in extending the database by applying all the possible modifications to each peptide of the base. However, this solution, leading to an exponential number of possibilities, is of course too time consuming [8]. The other one, SpectralAlignment $[1,9,10]$, is a dynamic programming algorithm that has been designed to identify peptides even in presence of modifications. This method works rather well for one or two modifications, but for a larger number of modifications, SpectralAlignment is not really sustainable [9].

Spectra comparison has an essential advantage, namely the precision in the comparison, allowing information to be drawn even from spectra which used to be unexploitable with a de novo approach. But spectra comparison has a major downfall: the running time, that is highly dependent on the protein database size used to infer theoretical spectra.

Given that the proteins we are trying to identify come from unsequenced species, the idea is to find similar proteins on phylogenetically related organisms. This is why our method will have to allow the correspondence of a spectrum with a slightly different peptide. Yet, we need results with few errors and for most spectra. Although de novo is specially designed to treat unsequenced species, it still leads to lots of misinterpreted or uninterpreted spectra. That is why the development of a new spectra comparison method tolerant to modifications appears interesting for us. 


\section{Definitions and Notations}

An MS/MS spectrum is obtained by selection, isolation and fragmentation of peptides within the mass spectrometer. Each peak results from the dissociation of a given peptide into two fragmented ions: an N-terminal one, and a C-terminal one. The $\mathbf{N}$-terminal ions (that are called $a, b, c$, see for instance Figure 1) represent the left part of the peptide sequence, and the C-terminal ions (that are called $x, y, z)$ represent the right part of the peptide sequence. The position where the fragmentation appears is located around the junction of two amino acids, as described in Figure 1. The $b$ and $y$ ions mark the exact junction point. In the rest of this paper, we will say that two different ions are dependent if they are issued from the fragmentation between the same two successive amino acids inside a peptide. For instance, in Figure 1 , for a given $i \in[1 ; 3], a_{i}, b_{i}, c_{i}$, $x_{i}, y_{i}$ and $z_{i}$ are mutually dependent ions. In a spectrum, a peak corresponding to a C-terminal (resp. N-terminal) ion is called C-terminal (resp. N-terminal). Moreover, peaks corresponding to dependent ions are called dependent peaks.

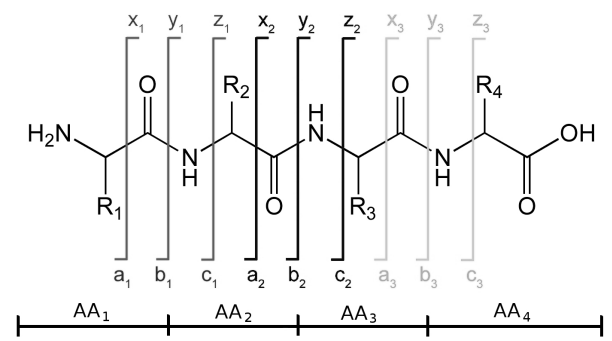

Fig. 1. This figure shows the fragmentation points inside a peptide containing four amino acids $\left(A A_{i}\right.$ with $\left.i \in[1 ; 4]\right) . R_{i}(i \in[1 ; 4])$ are chemical compounds that determine the corresponding amino acid. In this example, there are three sets of dependent ions.

We can notice that a symmetry exists between N-terminal and C-terminal peaks for a given fragmentation. In Figure 2 (a), the N-terminal peak located at position $m(G L)$ and the C-terminal peak located at position $m(M P R G)$ are linked by the relation $m(M P R G)=m(G L M P R G)-m(G L)-20(-20$ is due to the fact that the peptide is not symmetric at its extremities, see Figure 1, and to the ionization). This is a critical notion that is valid for any fragmentation and is seldom used. We call this relation inner symmetry.

Any spectrum produced by a mass spectrometer is called an experimental spectrum $\left(S_{e}\right)$, and any spectrum predicted in-silico from a peptide is called a theoretical spectrum $\left(S_{t}\right)$. In the following figures, spectra will always display all of the nine most frequent peaks coming from the fragmentation $[11,12]$.

Considering that the masses where the peaks appear can be seen as integers, we can represent a spectrum by a vector of booleans. This vector contains, for 


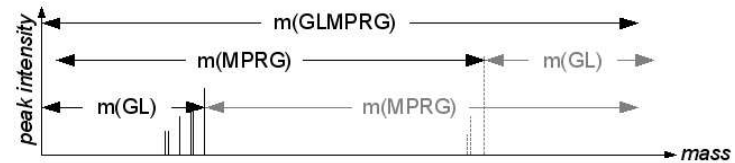

(a)

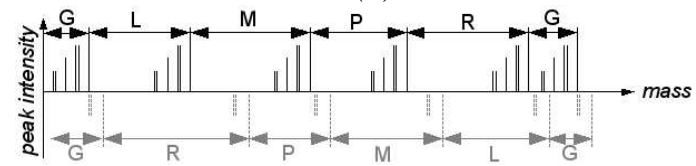

(b)

Fig. 2. (a) This piece of a spectrum shows the peaks created when the peptide GLMPRG (of mass $m(G L M P R G)$ ) is broken into peptides GL and MPRG. (b) is the spectrum of the peptide GLMPRG (for a better visualization, the N-terminal peaks are above the axis, the C-terminal ones are below the axis).

every mass, a boolean attesting the presence of a peak ('true') or its absence ('false'). Vector $V_{t}$ represents the spectrum $S_{t}$ and $V_{e}$ represents $S_{e}$. Then, as in the case of sequences, we can align the elements of $V_{t}$ and $V_{e}$ two by two while allowing the insertion of gaps. It is only necessary to ensure that both vectors have the same length and that a gap is not aligned with another gap. In this representation, a shift corresponds to the insertion of a gap in either $V_{t}$ or $V_{e}$, which itself corresponds to a peptide modification.

A score is used to evaluate an alignment, which represents the similarity between both spectra, in the sense that a higher score means a higher similarity. For instance, the number of common peaks in a given alignment is a possible score. When transposed to $V_{t}$ and $V_{e}$, this corresponds to the number of pairs of booleans in which both values are 'true' at the same position. In this context, the alignment having the highest score will be considered as the best alignment. Our goal is, given an experimental spectrum, to compare it to all the theoretical spectra from the database. For each comparison, we want to find the best alignment.

\section{Our Method}

Because we want to align a spectrum $S_{e}$ originated from unsequenced species with a spectrum $S_{t}$ generated from phylogenetically related organisms, our method must be able to take modifications into account. Here, modification means insertion, deletion or substitution of one or several amino acids, but we will see that our algorithm, by nature, is able to handle other types of modifications such as post translational modifications.

Dynamic programming is an appropriate algorithmic method to find the best alignment between two spectra, even in presence of modifications. It has been used in this context by methods such as SpectralAlignement (SA) [1]. 
For our method to be tolerant to substitutions, we must be careful about the way we shift peaks when we want to improve our alignment, because a substitution, by changing the mass of an amino acid, not only changes one peak, but also some other peaks inside the whole spectrum. To take into account a substitution, a naïve algorithm could simply shift all peaks positioned after the location of the substituted amino acid. However, this could drastically change the positions of the peaks in the whole spectrum, as shown in Figure 3. It can be seen that such a modification destroys the link between N-terminal and Cterminal peaks, causing the inner symmetry to be broken. Moreover, the loss of information due to this rupture in the symmetry grows with the number of modifications.

In a previous work [1], Pevzner et al. considered this notion of symmetry by proposing, for each peak of each spectrum, to add its symmetric twin peak. However, they noticed two major drawbacks to this:

- The addition of noise (the symmetric twin of a noise peak is another noise peak).

- During the alignment, if a peak $P$ (resp. a symmetric twin peak TP) is aligned, then $T P$ (resp. $P$ ) must not be used in the alignment. Deciding, for each peak of the spectrum, which one of these peaks $(P$ or $T P)$ must be aligned, is an NP-complete problem [11].

But it is important to note that the two spectra $S_{e}$ and $S_{t}$ do not present the same properties. By construction, in $S_{t}$, we can identify each peak as an Nterminal or a C-terminal peak. This extra information is fully exploited in our algorithm and eliminates both previously raised drawbacks.

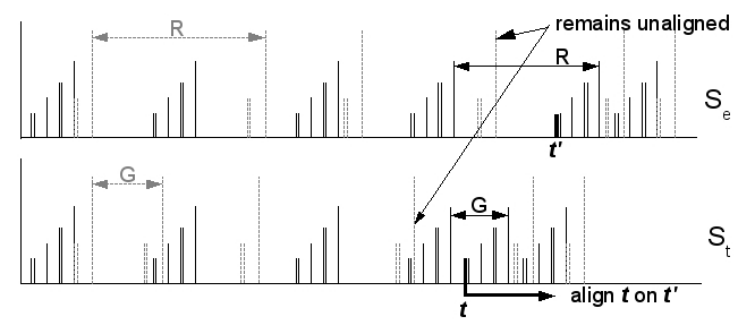

Fig. 3. In this figure, two spectra are represented. C-terminal peaks are dashed gray, Nterminal peaks are black. Spectrum $S_{e}$ represents the peptide GLMPRG, spectrum $S_{t}$ represents the peptide GLMPGG. This figure shows how one substitution in a peptide can highly change the contents of a spectrum (as it is, there are only 27 peaks aligned between $S_{e}$ and $S_{t}$ out of 54), and how shifting peaks from position $t$ to position $t^{\prime}$ is not sufficient to correctly align $S_{e}$ with $S_{t}$ (the black arrow shows the shift, resulting in an alignment of 45 peaks between $S_{e}$ and $S_{t}$ out of 54). Although the shift improves the alignment, some of the peaks remain unaligned after the shift has been applied. 


\subsection{Symmetry}

Theoretical Spectrum: We build the spectrum in-silico, so the location of all the different peaks is known. Considering this, we can easily remove all the Cterminal peaks and replace them by their respective symmetric peaks. Let $m_{i}$ be the mass of the $i$-th C-terminal peak; its mass will be replaced by $M_{\text {peptide }}-m_{i}$ where $M_{\text {peptide }}$ is the mass of the peptide represented in $S_{t}$. The theoretical spectrum, after the application of the symmetry, is called theoretical symmetric spectrum $\left(S S_{t}\right)$. Figure 4 (above) shows the spectrum $S_{t}$ corresponding to the peptide GLMPRG. Figure 4 (below) shows the spectrum $S S_{t}$, that is the spectrum $S_{t}$ to which the symmetry has been applied. Note that in $S S_{t}$, the distance between the N-terminal and C-terminal peaks from the same fragmentation is a constant (i.e. in spectrum $S S_{t}$ from Figure 4, peaks $t$ and $t^{\prime}$ (resp. $u$ and $u^{\prime}$ ) are dependent and the distance between $t$ and $t^{\prime}$ is the same than between $u$ and $u^{\prime}$ ), thus a shift of all the peaks positioned after a modification will still respect the inner symmetry. We also point out that our construction of $S S_{t}$ is different that the one proposed by Pevzner et al. in [1] on two points: (1) we do not apply symmetry on the N-terminal peaks and (2) when applying symmetry on C-terminal peaks, we remove the original peaks.

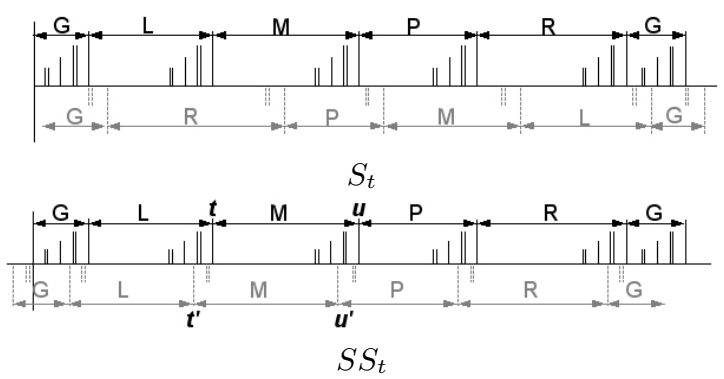

Fig. 4. $S_{t}$ (above) represents the spectrum before any symmetry is applied. $S S_{t}$ (below) represents the spectrum after the symmetry has been applied. In $S_{t}$ the dashed peaks are the N-terminal peaks. In $S S_{t}$ the dashed peaks are the N-terminal peaks to which symmetry has been applied.

Experimental Spectrum: As we do not know the ion type represented by each peak (in fact, a peak can represent the superposition of different ions of the same mass), we create a new symmetric peak for each existing peak of the spectrum. These peaks are created at position $M_{\text {peptide }}-m_{i}$, where $M_{\text {peptide }}$ represents the mass of the peptide measured by the mass spectrometer and $m_{i}$ the mass of the $i$-th peak of $S_{e}$. The experimental spectrum, after the application of the symmetry is called experimental symmetric spectrum $\left(S S_{e}\right)$. Figure 5 (above) shows the spectrum $S_{e}$ corresponding to the peptide $G L M P G G$. Figure 5 (below) shows the spectrum $S S_{e}$, that is the spectrum $S_{e}$ to which the symmetry has been applied. 
During the alignment, we need to forbid the alignment of some pairs of peaks. For instance, N-terminal peaks from $S S_{t}$ should be aligned with the original peaks from $S S_{e}$. This is important to guarantee that the solution is feasible. To do this, it is sufficient to keep track, for each peak, if it is a original peak or not.

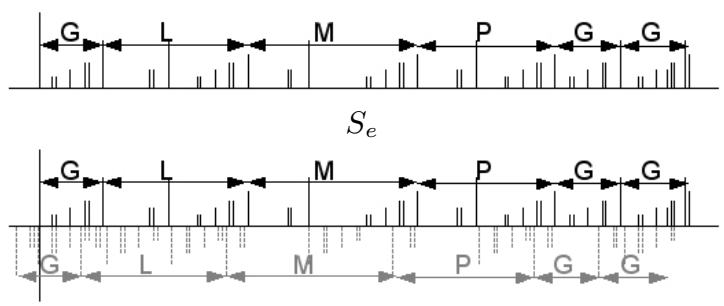

$S S_{e}$

Fig. 5. $S_{e}$ (above) represents the spectrum before any symmetry is applied. Then $S S_{e}$ (below) represents the spectrum after the symmetry has been applied. In $S S_{e}$, the dashed peaks are $S_{e}$ peaks to which symmetry has been applied.

\subsection{Packet}

The random fragmentation along the peptide backbone creates a number of different types of peaks. The location where the fragmentation occurs implies various peaks, $a, b, c$ and $x, y$, or $z$ (see Figure 1). Additionally, peaks corresponding to neutral loss (water, ammonia) are also frequently observed. For the construction of $S S_{t}$ we choose to keep, for each type of fragmentation, the nine most frequent peaks observed in experimental spectra when using a Quadrupole Time-of-Flight mass spectrometer [11,12] (see also Figure 6). After the application of symmetry, the notion of inner symmetry does not depend anymore of the peptide mass, thus these nine peaks may be clustered in a single packet. A packet represents all the fragmentations occurring between two consecutive amino acids of the peptide, thus $S S_{t}$ can now be represented by a group of packets. An example of packet is shown in Figure 6. Point $R_{p}$ marks the reference point of a packet $p$, and will be referred to when we will talk about a packet position. Introducing this notion of indivisible packet into the comparison between experimental and theoretical spectra allow us to forbid any translation that pulls apart dependent peaks: indeed, a shift can only exist between two packets.

In addition, to align $S S_{t}$ with $S S_{e}$, for each packet $p$ of $S S_{t}, R_{p}$ is positionned on a mass $m$ of $S S_{e}$. This gives an alignment of score $s$ between $p$ and $S S_{e}$. If $s$ goes past a threshold, then the mass $m$ is considered as one of the possible masses. In the rest of this paper, this score will be the number of aligned peaks. Increasing the threshold $T$ will speed up the alignment process because the number of possible masses will decrease (see Table 1). 
Another constraint which forbids the overlapping of two packets is added; it represents the fact that an amino acid has a minimum mass. That way, we do not allow the algorithm to make small and unrealistic shifts just to slightly improve its score (something which happens very often with SA).

Note that it is possible to modify the contents of a packet in order to adapt this notion for other types of mass spectrometers.

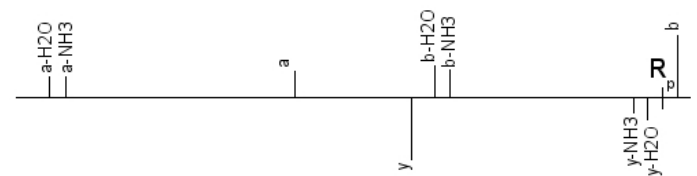

Fig. 6. The nine peaks representing the nine most current peaks that occur in MS/MS spectra ( $a, b, y$ and some of their variant peaks that are due to water or ammonia loss) result after the symmetry has been applied, to this packet. This packet is particularly suited for Quadrupole Time-of-Flight mass spectrometer.

\subsection{PacketSpectralAlignment Algorithm}

Our PacketSpectralAlignment (PSA) method needs three parameters: (i) $S S_{e}$ as described in Section 3.1, (ii) $S S_{t}$ for which the peaks are clustered into packets as described in Section 3.2 and (iii) $K$, the maximum number of allowed shifts. Our PSA algorithm (Algorithm 1) uses two matrices $M$ and $D$. The value $M[p][m][k]$ represents the best score obtained so far for the alignment of the peaks of the first packets of $S S_{t}$, up to packet number $p$, with all the first peaks from $S S_{e}$, up to the alignment of $R_{p}$, with mass $m$, and with at most $k$ shifts. The value $D[p][m][k]$ represents the best score of an alignment that must have $R_{p}$ aligned with mass $m$ in $S S_{e}$ and containing at most $k$ shifts. PSA will compute, for each possible number $k$ of shifts, each possible mass $m$ in $S S_{e}$ and for each packet $p$ of $S S_{t}$, the values $M[p][m][k]$ and $D[p][m][k]$.

The variable best contains the score of the best alignment met on the $D$ matrix that could be extended with the current position $(p, m, k)$ without more shifts (see Figure 7 for an illustration of how one of these alignments is found).

The Score function will return the score resulting from the alignment of the peaks of the $p$-th packet of $S S_{t}$ with the peaks of $S S_{e}$, when $R_{p}$ is positioned at the mass $m$ of $S S_{e}$.

The $D$ matrix is updated by choosing the best possibility between the two following cases:

a. we used the last value met on the diagonal, meaning no shift is needed, or

b. we must apply a shift and take the last score met on the diagonal in the $(k-1)$-th dimension of the matrix.

Then the $M$ matrix is updated by taking the best alignment found until this 
point.

As an illustration, applying our algorithm PSA on the theoretical spectrum $S S_{t}$ of Figure 4 and the experimental spectrum $S S_{e}$ of Figure 5 gives a "perfect" alignment of the peaks (i.e. that is, 54 peaks out of 54 that are aligned) with one shift (corresponding to the substitution of the fifth amino acid R by $\mathrm{G}$ in $S S_{t}$ ).
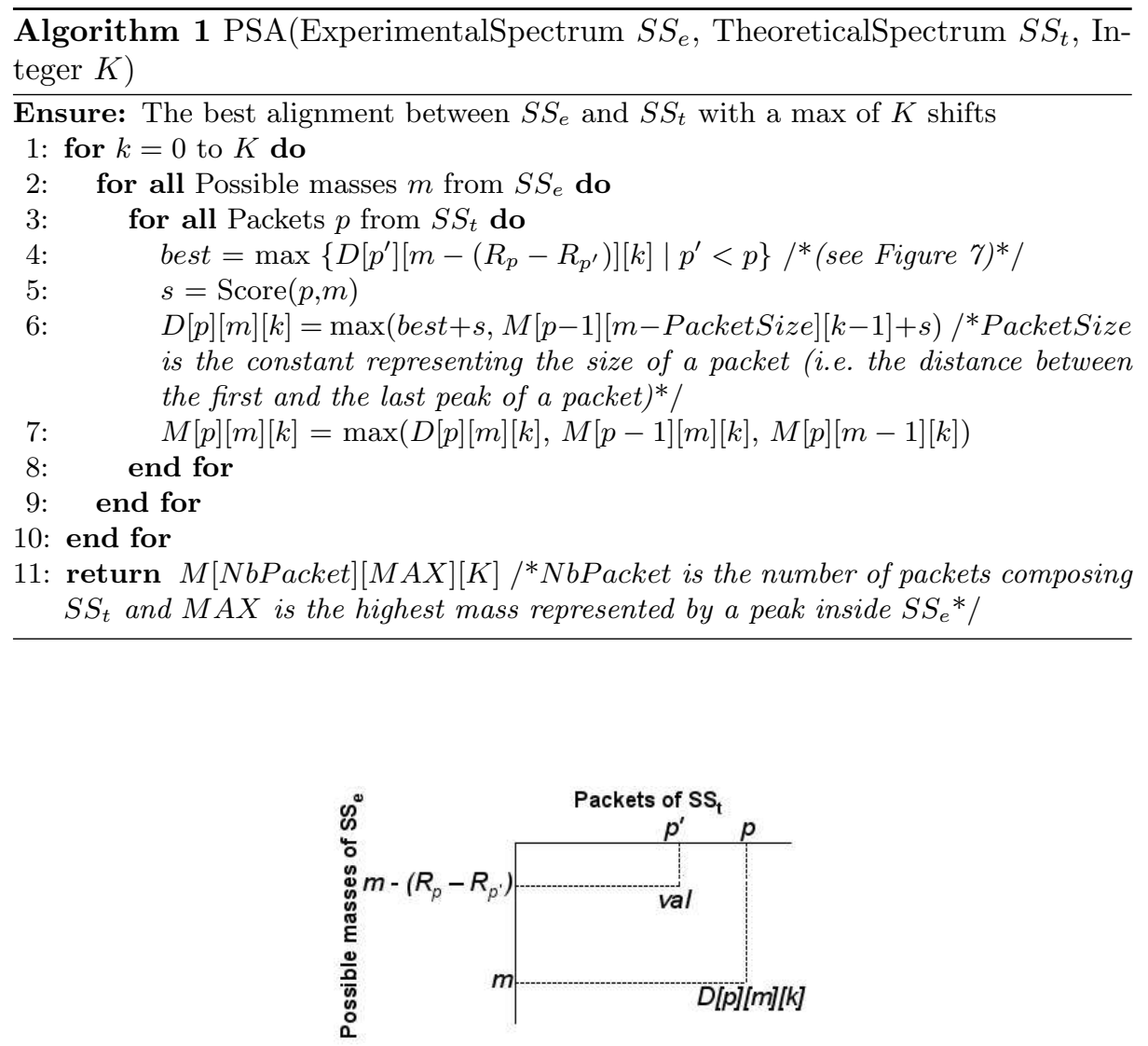

Fig. 7. This figure shows how to find a value val $=D\left[p^{\prime}\right]\left[m-\left(R_{p}-R_{p^{\prime}}\right)\right][k]$, with $p^{\prime}<p$.

\section{Results}

We compare our algorithm to $\mathrm{SA}$ on a set of simulated data. We generate a dataset of 1000 random peptides of random size in $[10,25]$ in order to constitute a database that will be used to create the theoretical symmetric spectra. Each 
peptide in the database is then modified in 5 different versions by applying 0 to 4 random substitutions of amino acids. These modified peptides are used to create 5 sets of artificial experimental symmetric spectra (one for each different number of modifications). These spectra are constituted using the nine most frequents peaks that are created considering the probability of apparition observed by Frank et al. [12]. Noise has been introduced in each spectra, adding $50 \%$ more peaks at random masses. All tests have been made using 1 dalton precision. For each $S S_{e}$, we call the target peptide of $S S_{e}$ (denoted $T P\left(S S_{e}\right)$ ) the original peptide sequence from the dataset that has been modified to obtain the spectrum.

Each $S S_{e}$ is compared with each $S S_{t}$. The score (here, the number of common peaks in the best alignment) resulting from each comparison is memorized and used to order the set of peptides for each $S S_{e}$. In the ideal case, for an experimental spectrum $S S_{e}, T P\left(S S_{e}\right)$ should obtain the highest score and thus should have rank one. Thus, by looking at the rank of the target, we can evaluate the capacity for PSA to take modifications into account. That is why we use the average rank of the target peptide, as well as the proportion of target peptides having rank one, as indicators to evaluate the two algorithms (as shown in Figure 8).
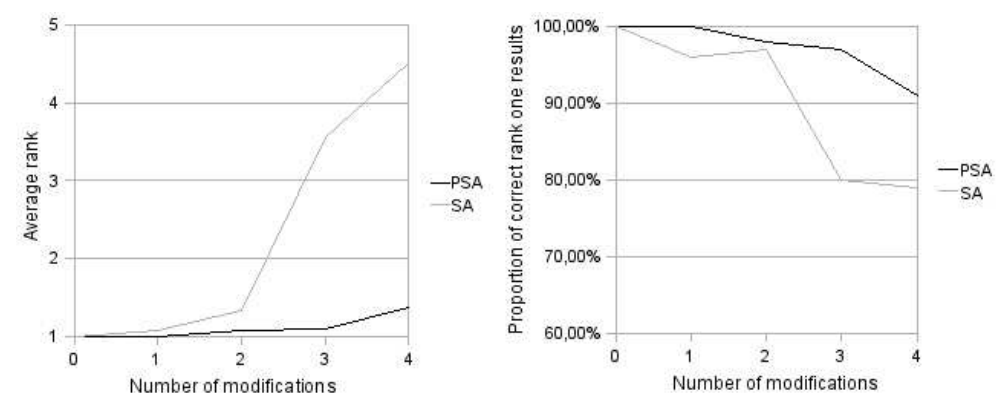

Fig. 8. Comparison of SA and PSA on our sets of 1000 random peptides

During the comparisons, the parameters used by SA and our algorithm PSA are the same. In particular, we use the same score function, which is the number of common peaks in both spectra. The number of shifts used by these two methods is set dynamically, depending on the size of the two compared spectra. We could have fixed this to a constant value, but allowing for instance $\frac{N}{2}$ shifts in an $N$ amino acids long peptide does not make any sense, so we chose to allow $k$ shifts for a peptide of mass $M$ where $k=\left\lceil\frac{M}{600}\right\rceil+1$. In the case of PSA, the threshold $T$ used to determine the possible masses kept in symmetric experimental spectra is set to 2 .

Our tests show that the two algorithms have a comparable behaviour for 0 to 2 shifts, with a slight advantage for our algorithm. However, for more than two 
shifts, SpectralAlignment presents a fast deterioration of its results, while PacketSpectralAlignment still gives good results (see Figure 8). We also note that on these tests, for a threshold $T$ of 2, our algorithm PSA is twice as fast as SA.

We have also evaluated the benefits supplied by the packets, and more particularly by the number of possible masses. As explained in Section 3.2, we do not test all masses in $S S_{e}$, but only those masses $m$ inducing an alignment of at least $T$ peaks when the reference point $R_{p}$ of a packet $p$ from $S S_{t}$ is positionned at mass $m$. To evaluate this, we have computed the number of possible masses for different values of $T$ on four different datasets. The first one is a set of 1000 simulated spectra of size $[10,25]$ with $50 \%$ of noise peaks, generated the same way as described at the beginning of Section 4 . On this dataset, a spectrum contains on average 150 peaks. Then we use three sets of 140 experimental maize spectra on which we apply different filters: (1) we keep all peaks (meaning an average of 275 peaks per spectrum), (2) we keep the 100 most intense peaks, and (3) we keep the 50 most intense peaks. Table 1 shows the evolution of the number of possible masses in function of the threshold $T$ for each set of spectra. We can notice that the number of possible masses decreases considerably when $T$ is increased.

\begin{tabular}{|l|l|c|c|c|c|}
\hline \multicolumn{5}{|c|}{ Number of Possible Masses } \\
\hline \multicolumn{2}{|c|}{} & \multicolumn{4}{|c|}{ Threshold T } \\
\cline { 3 - 6 } & & 1 & 2 & 3 & 4 \\
\hline Simulated spectra & 485 & 134 & 39 & 14 \\
\hline \multirow{3}{*}{ Experimental Maize spectra } & no filtering & 689 & 312 & 141 & 61 \\
\cline { 2 - 6 } & 100 most intense peaks & 540 & 180 & 57 & 17 \\
\cline { 2 - 6 } & 50 most intense peaks & 346 & 79 & 18 & 4 \\
\hline
\end{tabular}

Table 1. Evaluation of the number of possible masses on four sets of spectra depending on the threshold $T$.

\section{Conclusion}

We have developed PacketSpectralAlignment, a new dynamic programming based algorithm that fully exploits, for the first time, two properties that are inherent to MS/MS spectra. The first one consists in using the inner symmetry of spectra and the second one is the grouping of all dependent peaks into packets. Although our algorithm was at first motivated by the identification of proteins in unsequenced organisms, it does not set any constraints on the allowed shifts in the alignment. Thus, PSA is also able to handle the discovery of post translational modifications.

Our results are very positive, showing a serious increase in peptides identification in spite of modifications. The sensibility has been significantly increased, 
while the execution time has been divided by more than two. More tests on experimental data will allow us to evaluate more precisely the benefits provided by our new algorithm. In the future, a better consideration of other points, such as spectra quality, will be added. Moreover, the score will be improved by taking into account other elements such as peaks intensity.

Acknowledgments. MS/MS experimental spectra were performed with the facilities of the platform Biopolymers, Interactions and Structural Biology, INRA Nantes. The authors thank Dr Hélène Rogniaux for fruitful discussions about MS/MS spectra interpretation. This research was supported by grant from the Region Pays de la Loire, France.

\section{References}

1. Pevzner, P.A., Dancík, V., Tang, C.L.: Mutation-tolerant protein identification by mass spectrometry. J Comput Biol 7(6) (2000) 777-87

2. Habermann, B., Oegema, J., Sunyaev, S., Shevchenko, A.: The power and the limitations of cross-species protein identification by mass spectrometry-driven sequence similarity searches. Mol Cell Proteomics 3(3) (2004) 238-49

3. Grossmann, J., Fischer, B., Baerenfaller, K., Owiti, J., Buhmann, J.M., Gruissem, W., Baginsky, S.: A workflow to increase the detection rate of proteins from unsequenced organisms in high-throughput proteomics experiments. Proteomics 7(23) (2007) 4245-54

4. Pevtsov, S., Fedulova, I., Mirzaei, H., Buck, C., Zhang, X.: Performance evaluation of existing de novo sequencing algorithms. J Proteome Res 5(11) (2006) 3018-28

5. Pitzer, E., Masselot, A., Colinge, J.: Assessing peptide de novo sequencing algorithms performance on large and diverse data sets. Proteomics 7(17) (2007) 3051-4

6. Eng, J., McCormack, A., Yates, J.: An approach to correlate tandem mass spectral data of peptides with amino acid sequences in a protein database. J. Am. Soc. Mass Spectrom 5(11) (1994) 976-989

7. Perkins, D.N., Pappin, D.J., Creasy, D.M., Cottrell, J.S.: Probability-based protein identification by searching sequence databases using mass spectrometry data. Electrophoresis 20(18) (1999) 3551-67

8. Yates, J.R., Eng, J.K., McCormack, A.L., Schieltz, D.: Method to correlate tandem mass spectra of modified peptides to amino acid sequences in the protein database. Anal Chem 67(8) (1995) 1426-36

9. Pevzner, P.A., Mulyukov, Z., Dancik, V., Tang, C.L.: Efficiency of database search for identification of mutated and modified proteins via mass spectrometry. Genome Res 11(2) (2001) 290-9

10. Tsur, D., Tanner, S., Zandi, E., Bafna, V., Pevzner, P.A.: Identification of posttranslational modifications by blind search of mass spectra. Nat Biotechnol 23(12) (2005) 1562-7

11. Dancik, V., Addona, T., Clauser, K., Vath, J., Pevzner, P.: De novo peptide sequencing via tandem mass spectrometry. Journal of Computational Biology 6(3-4) (1999) 327-342

12. Frank, A.M., Savitski, M.M., Nielsen, M.L., Zubarev, R.A., Pevzner, P.A.: De novo peptide sequencing and identification with precision mass spectrometry. J Proteome Res 6(1) (2007) 114-23 